REVISTA CIENCIAS BIOMÉDICAS

PRESENTACIÓN DE CASOS CLÍNICOS

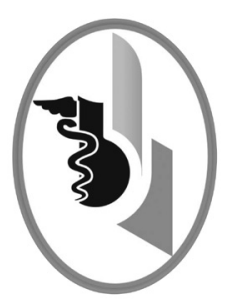

\title{
GANGRENA DE FORUNIER: MANEJO CON APÓSITO DE CARBOXIMETILCELULOSA Y PLATA IÓNICA
}

\author{
FOURNIER GANGRENE: MANAGEMENTWITH \\ CARBOXYMETHYLCELLULOSE AND SILVER DRESSINGS
}

Osorio-Lambis Martha ${ }^{1}$

Múnera-Bohórquez Ulises²

Porto-Osorio Leidy ${ }^{3}$

Correspondencia: mosoriol1@unicartagena.edu.co

Recibido para evaluación: junio - 17 - 2015. Aceptado para publicación: febrero - 25 - 2016.

\section{RESUMEN}

Introducción: la gangrena de Fournier (GF) o fascitis necrotizante es una patología infecciosa, amenazante para la vida, fulminante y progresiva que afecta genitales externos y periné. Después del desbridamiento se requiere de cuidados especializados y avanzados en el manejo de las heridas para reducir la morbilidad y mortalidad.

Objetivo: presentar un caso clínico de un paciente con diagnóstico de GF que recibió cuidados domiciliarios de sus heridas con apósitos medicados con carboximetilcelulosa y plata iónica en la ciudad de Cartagena- Colombia.

Caso clínico: paciente masculino de 58 años de edad con antecedentes de diabetes mellitus e hipertensión arterial, quien presentó absceso perianal. Fue tratado inicialmente con antibióticos orales sin mejoría, posteriormente, se le diagnosticó GF. Requirió manejo hospitalario con antibioticoterapia intravenosa biconjugada y desbridamiento quirúrgico. Fue removida la piel escrotal en su totalidad hasta dejar expuesta la fascia cremastérica desde la base del escroto hasta la región perianal de ambos lados de la línea media. Recibió cuidados médicos domiciliarios y de enfermería de sus heridas en un ambiente húmedo con apósitos de carboximetilcelulosa y plata iónica hasta su cicatrización total. Procedimiento realizado cada tercer día, un total de doce curaciones (36 días). Se evidenció cicatrización total de las heridas sin complicaciones.

Conclusión: los resultados obtenidos con la aplicación de este tratamiento fueron muy buenos, ya que se logró la cicatrización total de las lesiones sin intervenciones quirúrgicas adicionales. Rev.cienc.biomed. 2016;7(1):133-138.

\section{PALABRAS CLAVE}

Gangrena de Fournier, Fascitis necrotizante; Cicatrización de heridas.

\footnotetext{
Enfermera. Especialista Medicoquirúrgica. Magíster en Enfermería. Docente. Facultad de Enfermería. Universidad de Cartagena. Colombia. Médico. Especialista en Salud Ocupacional. Magíster en Administración. SIME SAS. Cartagena. Colombia.

Enfermera. SIME SAS. Cartagena. Colombia.
} 


\section{SUMMARY}

Introduction: Fournier gangrene (FG) or necrotizing fasciitis is a life-threatening infectious pathology, fulminant and progressive that affects external genitals and perineum. It is required specialized and advanced care in the management of heals to reduce morbidity and mortality after debridement.

Objective: to present a case record about a male patient with FG that received home care of his heals with carboxymethylcellulose and silver dressings in Cartagena-Colombia. Case report: it is presented the case of a 58-year-old male patient with a history of diabetes mellitus and hypertension, who presented a perianal abscess. He was initially treated with oral antibiotics without improvement, subsequently, he was diagnosed with FG. He required hospital management with biconjugated intravenous antibiotic therapy and surgical debridement. The entire scrotal skin was removed until the cremasteric fascia was exposed from the base of the scrotum to the perianal region on both sides of the midline. He received home and nursing care from his wounds in a humid environment with carboxymethylcellulose and silver dressings until their total healing. Procedure performed every third day, a total of 12 cures (36 days). It was evidenced a complete healing of the wounds without complications.

Conclusion: the results obtained with the application of this treatment were very good because there was a total scarring of the wounds without additional surgical interventions. Rev.cienc.biomed. 2016;7(1):133-138.

\section{KEYWORDS}

Fournier gangrene; Necrotizing fasciitis; Wound healing.

\section{INTRODUCCIÓN}

La gangrena de Fournier (GF), también conocida como fascitis necrotizante, gangrena estreptocócica, gangrena idiopática o flemón perineal, es una patología infecciosa, genitourinaria causada principalmente por una flora polimicrobiana, debida a bacilos gram-negativos como la Escherichia coli, Klebsiella, Proteus, Enterobacter y anaerobios propios del área perineal que proceden del colon. Amenazante para la vida, fulminante y progresiva, acompañada de necrosis de la piel y tejidos blandos con elevada morbilidad y mortalidad, llegando a ser del $20-80 \%$. Afecta genitales externos y periné con incidencia mayor en el sexo masculino aunque puede también afectar a mujeres $(1,2,3)$.

La GF fue referida por Baurienne en 1764, $\mathrm{y}$, posteriormente, por A. L. Fournier en 1883 (4). Su incidencia es de 1 en 7.500 casos, en edades que oscilan entre 40-70 años (5). El diagnóstico es clínico, basado en una historia clínica completa que incluye antecedentes y síntomas (3). Dentro de los factores de riesgos están la edad avanzada, la presencia de una enfermedad de base que afecte la microcirculación (diabetes mellitus 20-66\%, enfermedad vascular periférica), alcoholismo 20-60\%, obesidad, cáncer, insuficiencia renal crónica, estenosis uretral con extravasación proximal, traumatismos recientes en el periné, fístulas y abscesos perianales y cualquier proceso que afecte el estado inmunitario en general (3).

La GF se caracteriza por un inicio abrupto de una infección necrotizante de los tejidos blandos de la región genital y perirectal, esta involucra las fascias profundas con velocidad de diseminación de la necrosis de $2-3 \mathrm{cms} / \mathrm{h}$ (3). El desbridamiento quirúrgico inmediato con escisión de todo el tejido necrótico que debe superar las áreas comprometidas hasta hallar la fascia normal, acompañado de tratamiento agresivo con antibióticos para reducir las tasas de morbilidad y mortalidad (6).

La GF suele dejar lesiones con amplia pérdida de tejidos que amerita manejo especializado y abordaje adecuado para lograr la recuperación de los mismos. Se han utilizado apósitos con alginato de calcio, antimicrobianos tópicos, apósitos de celulosa (7-10). El éxito de la curación de los pacientes con GF requiere de la vigilancia y continuidad en el tratamiento del lecho de la lesión para así contribuir a una rápida recuperación de la integridad cutánea (7). 
Singh R. (7) y Jurczak F. (8) reportaron el potencial antiséptico de los apósitos con plata iónica en heridas crónicas, quemaduras y úlceras por decúbito que ayudan a combatir infecciones cuando están presentes o a prevenirlas. Los apósitos con plata iónica tienen actividad antimicrobiana para Pseudomona aeruginosa, Staphylococcus aereus, Bacteroides fragilis, Klebsiella pneumoniae, Enterococcus, Eschericha coli e incluso hongos como Candida albicans. Igualmente, Singh R. sostiene que una adecuada cicatrización disminuye el número de requerimientos de lavados quirúrgicos (7).

Por otro lado, Cooper M. (11) describe que existe un gran número de preparados tópicos que han demostrado ser eficaces en la reducción de los recuentos bacterianos en heridas, entre ellos el ácido acético, el cloranfenicol y las soluciones yodadas como tóxicos para los fibroblastos y queratinocitos. El objetivo es presentar un paciente con diagnóstico de GF que recibió cuidados domiciliarios de sus heridas con apósitos medicados con carboximetilcelulosa y plata iónica en la ciudad de Cartagena de Indias - Colombia.

\section{CASO CLÍNICO}

Paciente masculino de 58 años de edad con diagnóstico de diabetes mellitus e hipertensión arterial controlada, quien presentó absceso perianal que fue tratado inicialmente con antibioticoterapia oral sin mejoría. Posteriormente, presentó olor fétido en región genital y fiebre alta, motivo por el cual fue trasladado a un centro hospitalario en el que fue internado. En el momento del examen presentaba estado febril con deshidratación y olor fétido en región perianal, se identificó zona abscedada con eritema y edema de la piel con crepitación subcutánea que se extendía a escroto y región perineal. Se le diagnosticó GF y sepsis de tejidos blandos, luego se inició antibioticoterapia intravenosa biconjugada y fue llevado a cirugía para desbridamiento quirúrgico de tejidos blandos en región genital y adyacente. Durante el acto quirúrgico presentó arritmia que fue manejada exitosamente intraoperatoriamente. Le fue removida la piel escrotal en su totalidad hasta dejar expuesta la fascia cremastérica y todo et tejido desvitalizado perineal, desde la base del escroto hasta la región perianal a ambos lados de la línea media. Durante su estancia en UCI, la zona expuesta fue manejada con métodos tradicionales de curación, con lo que presentó mejoría de su estado general. Ulteriormente, se solicitó evaluación y cuidados por clínica de heridas complejas.

En la valoración inicial por clínica de heridas se encontró herida grado II, extensa en región escrotal con exposición de fascia cremastérica y compromiso de la región perineal, en fase de granulación activa (40\%), con restos de fibrina $(60 \%)$ y exudado serosanguinolento en moderada cantidad. Se solicitó consentimiento informado para iniciar los cuidados de sus heridas y para la publicación de los resultados.

Se realizaron curaciones en ambiente húmedo, previa irrigación con solución salina normal para quitar los apósitos colocados en la curación anterior, el exudado se retiraba con el dedo enguantado y solución salina normal a chorro. Posteriormente, se colocaron apósitos medicados de carboximetilcelulosa y plata iónica en todas las lesiones acompañados de cobertura con apósito hidropolímero para regular el exudado y aislar la herida de la zona perineal. Por la ubicación de las lesiones y los riesgos de contaminación, se realizó curación y cambio de apósitos medicados cada tercer día hasta la cicatrización total. En la segunda y tercera curación se evidenció desbridamiento autolítico de la fibrina, herida granulada en un $100 \%$ y borde con epitelización activa.

En cada procedimiento las heridas se encontraron sin evidencia de infección. Adicionalmente, el paciente se realizaba glucometría diaria en ayunas, obteniendo resultados dentro de los parámetros normales. La secuencia fotográfica permite apreciar la evolución de las lesiones y la cicatrización total alcanzada a los 36 días de estar recibiendo los cuidados de curaciones en ambiente húmedo (Figuras $\mathrm{N}^{\circ} 1,2,3,4$ ). Se brindó orientación y educación al paciente y familiares sobre las características del método de curaciones y los cuidados relacionados con la higiene del paciente. 


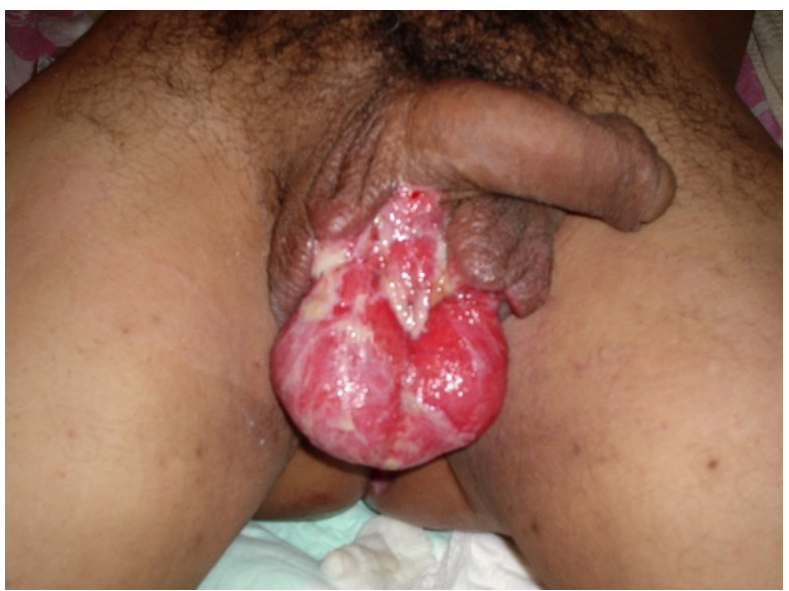

Figura No 1.

Lesión grado II, extensa con pérdida total de piel escrotal, compromiso de región perineal, granulación activa y el $70 \%$ de la superficie de la herida con tejido fibrinoide.

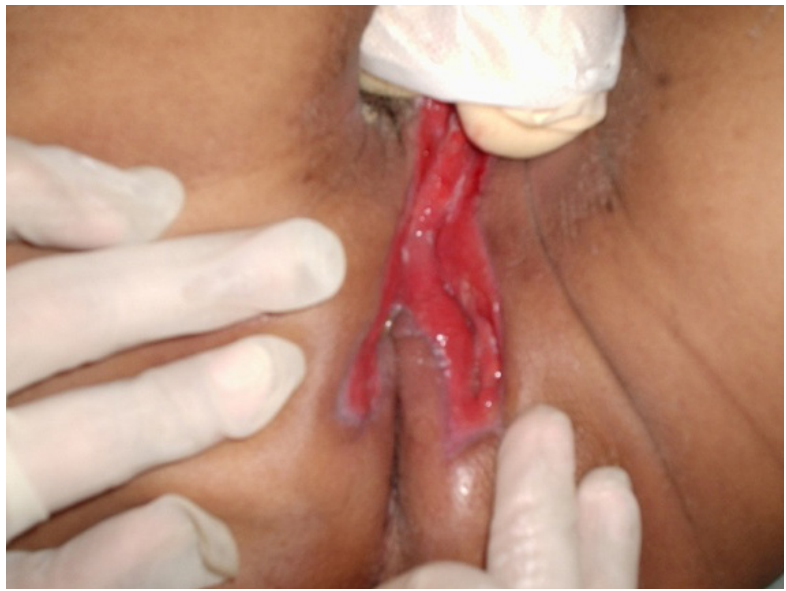

Figura No 2.

Lesión grado II y III cavitada, con compromiso de región perianal, granulación activa en 100\%.

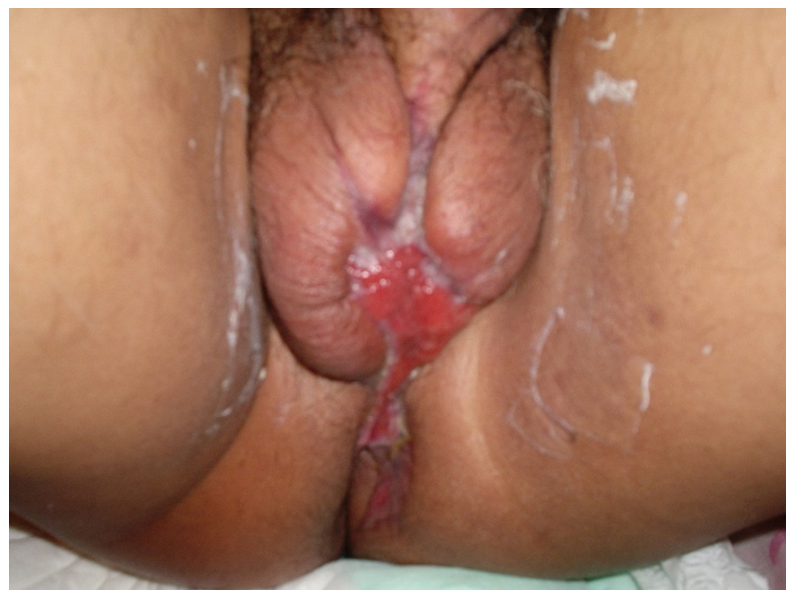

Figuras No 3.

Herida en fase de granulación total y contracción, bordes con epitelización activa.

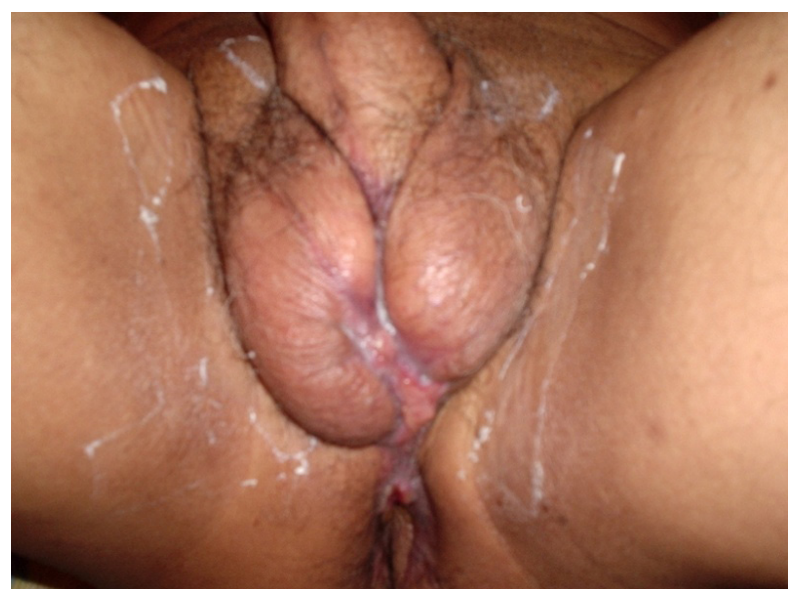

Figura No 4.

Herida cicatrizada por segunda intención.

\section{DISCUSIÓN}

El proceso de cicatrización de las heridas es un proceso dinámico y complejo, en el que inciden mecanismos fisiológicos sincronizados e interdependientes que ponen en marcha el organismo para reconstruir los tejidos lesionados. Puede prolongarse desde semanas hasta meses, e incluso años de acuerdo a las condiciones de cada situación $(12,13)$.

Los antecedentes como la diabetes, características de la herida (proceso infeccioso) y ubicación de la lesión son reportados por la literatura como factores directos e indirectos que pueden incidir en el proceso de cicatrización normal de una herida $(1,2,3,13)$. Por otro lado, Torra $]$. (13) describe que el exceso de humedad o viceversa ralentiza el proceso de cicatrización y constituye un factor de riesgo que puede desarrollar un proceso infeccioso por ser un medio ideal para la proliferación bacteriana.

Los apósitos antimicrobianos tópicos con plata son útiles en el tratamiento de las heridas con carga bacteriana elevada o con signos de infección precoz localizada. La plata interfiere en el metabolismo de las bacterias, rompe la pared celular bacteriana y se une al ADN inhibiendo la replicación y la probabilidad de desarrollar resistencia. Ejerce efectos antiinflamatorios favoreciendo la formación de vasos sanguíneos (angiogénesis) (14). Su selección dependerá del estado de la herida, 
la cantidad de exudado y adaptabilidad del apósito a la herida $(15,16)$.

Teniendo en cuenta los factores anteriormente descritos que, presentaba el paciente en estudio, y, considerando que el cuidado ideal de una herida debe promover el cierre completo de la lesión o la preparación de esta para un cierre por cuarta intención (17), se seleccionó para brindar los cuidados en este caso, el apósito de carboximetilcelulosa y plata iónica. Su acción antimicrobiana permitió el control sostenido de las bacterias propias de la región perineal. Por otro lado, la capacidad de absorción del exudado proporcionó un ambiente húmedo a la herida favoreciendo la migración celular. Su efecto antiinflamatorio propició la angiogénesis y se observó granulación en un $100 \%$ a partir de la segunda y tercera curación, y la cicatrización en un menor tiempo. Igualmente, el ambiente húmedo favoreció el desbridamiento autolítico de los restos de fibrina evidenciados en la valoración inicial.

La opción de recibir cuidados de las lesiones en casa por profesionales entrenados permitió el seguimiento, control de la lesión y acompañamiento al paciente hasta su cicatrización total sin complicaciones e intervenciones quirúrgicas adicionales.

Los resultados de este caso en lo referente al promedio de edad difieren de lo reportado por Barreda (18), Navarro J. (19), Navarro V.
(20) y Puerto P. (21), quienes describen un promedio de edades de 60,50, 66 y 66 años respectivamente. El antecedente personal de diabetes ratifica lo reportado por Puerto (20) y Medina P. (22).

La cicatrización de las lesiones con apósitos biotecnológicos avanzados comprueba lo reportado por Gago M. (23), quien refiere tres casos de pacientes con GF a quienes la cura húmeda sobre pérdida de sustancia utilizando carboximetilcelulosa y plata iónica en el proceso de cicatrización se dio sin ninguna complicación. Uno de ellos presentó cierre por tercera intención.

\section{CONCLUSIÓN}

Los cuidados en ambiente húmedo con apósitos de carboximetilcelulosa y plata iónica son una opción válida para el cuidado de las heridas en pacientes con GF, ya que permitieron el cierre total de las lesiones por segunda intención en doce curaciones, sin intervenciones quirúrgicas adicionales ni complicaciones en el paciente.

CONFLICTO DE INTERESES: ninguno que declarar.

FINANCIACIÓN: recursos propios de los autores. Honorarios, material quirúrgico e insumos hospitalarios aportados dentro de la atención asistencial del paciente.

\section{REFERENCIAS BIBLIOGRÁFICAS}

1. Urdaneta C E, Méndez P A, Urdaneta C A. Fournier: Perspectivas actuales. An. Med. Interna (Madrid) [revista en la Internet]. 2007; 24(4): 190-194.

2. Planelles G J, Vergés P A, Rubio T I, Beltrán A J, Carrascosa L V, San Juan de L C. Gangrena de Fournier. Arch. Esp. Urol. 2006; 59(8): 767-771.

3. Torres M L. Tratado de cuidados críticos y emergencias. Tomo II. España. 2002. 1348-1352.

4. Alvarado A, Bósquez A. Fascitis necrotizante. Revista Médico Científica. 2012; 25(2):24-5.

5. Valaguez VC, Rodríguez GA, Arenas OJ. Gangrena de Fournier en el Hospital de Infectología del Centro Médico Nacional La Raza. Educ Invest Clin. 2001;2(1):18-25.

6. Campbell-W. Urología. Tomo I. Editorial Médica Panamericana. Novena edición. 2008; 301-303.

7. Singh R, Kumar D, Kumar P, Chacharkar M. Development and evaluation of silver-impregnated amniotic membrane as an antimicrobial burn dressing. J Burn Care Res 2008; 29: 64-72.

8. Jurczak F, Dugre T, Johnstone A, Offori T, Vujovic Z, Hollander D; AQUACEL Aq Surgical/ Trauma Wound Study Group. Randomized clinical trial of hydrofiber dressing with silver versus povidone-iodine gauze in the management of open surgical and traumatic wounds. Int Wound J 2007; 4: 66-76.

9. Castellano J J, Shafii SM, Ko F, Donate G, Wright TE, Mannari RJ, et al. Comparative evaluation of silver-containing antimicrobial dressings and drugs. Int Wound J 2007; 4: 114-122.

10. Alonso T M., Buil B G., Fuenmayor D A., Laguna R E., Sandino M E. Análisis descriptivos de tratamientos tópicos en gangrena de Fournier. Asociación Española de Enfermería en Urología. $2011 ; 120: 18-22$. 
11 Cooper ML, Laer JA, Hansbrough JF. The cytotoxic effects of commonly used topical antimicrobial agents on human fibroblast and keratinocytes. Trauma. Recuperado de http:// www.ncbi.nlm.nih.gov.

12 Benítez G Y, Sagó M J, Pupo R A, Fernández C M, Infante P E. Presentación de un paciente con gangrena de Fournier. CCM. 2015; 19(3). 556-564.

13 Torra J E, Martínez C F, García F F, Rovira C G, Segovia G T. El proceso biológico de reparación de las heridas. En: Atención integral de las heridas crónicas segunda adicción. Londoño: GNEAUPP-FSJJ. 2016.

14. Consenso Internacional. Uso adecuado de los apósitos de plata en las heridas. Consenso del grupo de trabajo de expertos. London: Wounds Internacional. 2012. Disponible en http:// www.woundsinternational.com/media/issues/591/files/content_10498.pdf.

15. Soldevilla J. Tratamiento de la infección en heridas. Documento de posicionamiento GNEAUPP. 2006.

16. Sibbald RG, Contreras-RJ, Coutts P, Fierheller M, Rothman A, Woo K. Bacteriology, inflammation, and healing: a study of nanocrystalline silver dressings in chronic venous leg ulcers. Adv Skin Wound Care 2007; 20: 549-558.

17. Flores M I. Manejo avanzado de heridas. Revista Mexicana de Enfermería Cardiológica. 2006; 14(1). 24-28.

18. Barreda T J, Millán M, Suárez C, Cuadrado J, Rodríguez J, Franco E, Biondo S. Gangrena de Fournier. Estudio retrospectivo 41 casos. Rev. Cirugía Española. 2010. 87(4). 218-223.

19. Navarro J. Gangrena de Fournier. Rev. Eviden Invest Clinic.2010;3 (1): 51-7.

20. Navarro V, Salavert M, Campo C. Gangrena de Fournier estudio de 12 pacientes y revisión. Revista Española de Quimioterapia.2000;13(2):20-9.

21. Puerto P A, García S E, Morilla G M, Romero R M, Bravo S C, Urbina P A, Machado F G, Ramos M J, García G A. Gangrena de Fournier: Análisis descriptivo muestra serie 26 pacientes. Revista Argentina de Urología. 2013;78(3): 90-94.

22. Medina P J, Tejido S A, De la Rosa K F, Felip S N, Blanco Á M, Leiva G O. Gangrena de Fournier: Estudio de los factores pronósticos en 90 pacientes. Actas Urol Esp. 2008; 32(10):1024-30.

23. Gago M, Rodríguez $M L$, Diez $R$, Fernández $\mathrm{H}$. Cura húmeda sobre pérdida de sustancia en gangrena de Fournier. Asociación Española de Enfermería en Urología. 2010; 114: 21-23.

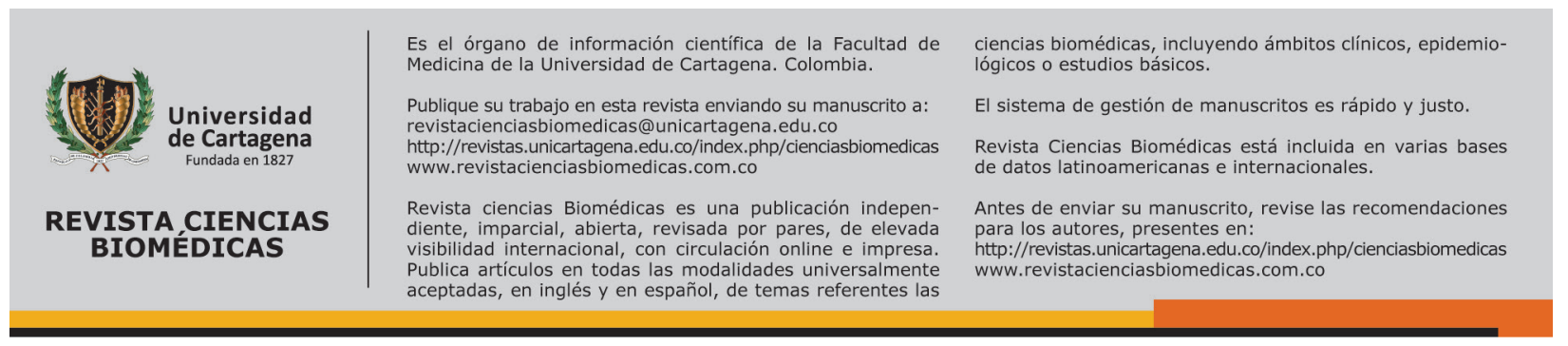

